\title{
Phase Behavior and Structure of a New Colloidal Model System of Bowl-Shaped Particles
}

\author{
Matthieu Marechal, Rob J. Kortschot, ${ }^{\dagger}$ Ahmet Faik Demirörs, Arnout Imhof, and \\ Marjolein Dijkstra*
}

Soft Condensed Matter, Debye Institute for NanoMaterials Science, Utrecht University, Princetonplein 5, 3584 CC Utrecht, The Netherlands

\begin{abstract}
We study the phase behavior of bowl-shaped (nano)particles using confocal microscopy and computer simulations. Experimentally, we find the formation of a wormlike fluid phase in which the bowl-shaped particles have a strong tendency to stack on top of each other. However, using free energy calculations in computer simulations, we show that the wormlike phase is out-ofequilibrium and that the columnar phase is thermodynamically stable for sufficiently deep bowls and high densities. In addition, we employ a novel technique based on simulated annealing to predict the crystal structures for shallow bowls. We find four exotic new crystal structures and we determine their region of stability using free energy calculations. We discuss the implications of our results for the development of materials consisting of molecular mesogens or nanoparticles.
\end{abstract}

KEYWORDS Nanobowls, crystal structures, computer simulations, phase diagram, columnar liquid crystals

$\tau$ n n recent years, a whole variety of bowl-shaped nanoparticles and colloids have been synthesized and characterized, ${ }^{1-6}$ and possible applications of these systems have been put forward. Most of these applications are of a single particle nature, such as a nanocontainer, ${ }^{4}$ or for metallic nanobowls depend on the tendency of these particles to form foamlike structures upon aggregation. Applications of the latter kind include superhydrophobic ${ }^{5}$ and infrared-blocking ${ }^{3}$ coatings. Metallic nanoparticles can be stabilized, for example, by applying a capping layer, ${ }^{7}$ which should prevent aggregation and thus reveal the natural tendency of bowl-shaped particles to form stacks or columns. In the molecular liquid crystal community, this tendency has been thought to decrease the number of defects in columnar phases, which is important if these columnar phases are to replace ${ }^{8}$ the crystalline ferroelectrics (materials with a permanent electric polarization) in (future) applications, such as sensors, electromechanical devices, and nonvolatile memory. ${ }^{9}$ Several bowl-shaped molecules have already been synthesized and found to form columnar phases. ${ }^{10}$ In addition, buckybowlic molecules, that is, fragments of $C_{60}$ whose dangling bonds have been saturated with hydrogen atoms, have been shown to crystallize in a columnar fashion. ${ }^{1-15}$ An aligned phase of metallic nanobowls could also be a promising novel material, since the individual particles have strongly anisotropic optical properties. ${ }^{1,3}$ However, no systematic experimental studies of the struc-

\footnotetext{
* To whom correspondence should be addressed. E-mail: M.Dijkstra1@uu.nl

${ }^{\dagger}$ Currently at the Physical and Colloid Chemistry group at Utrecht University. Received for review: 03/4/2010

Published on Web: 04/13/2010
}

ture of nanobowls in solution exist to our knowledge, and therefore it remains an open question whether or not bowlshaped nanoparticles and colloids can form stacks and ordered structures. This issue is also not easily resolved using theory or simulations as it is difficult to model the complicated particle shape, although a recent simulation study ${ }^{8}$ exists in which the attractive-repulsive Gay-Berne potential was generalized to a bowl-shaped particle. In another very recent simulation study ${ }^{16}$ of hard contact lenses (infinitely thin, shallow bowls), no columnar phase was found since the focus lay on rather shallow bowls at a relatively low densities.

In this letter, we study whether the formation of stacks and columnar liquid crystalline phases can be driven by entropy alone, that is, without any attractive interactions. To this end, we present confocal microscopy experiments on a new colloidal model system of purely repulsive bowlshaped colloids, which can be synthesized in the bulk (contrary to most nanobowls) and allow real-space observations in situ. Additionally, we performed computer simulations in which the stacking and phase behavior of hard bowlshaped particles are investigated as a function of the thickness (or equivalently deepness). We show that bowls form stacks and ordered structures, but the self-organization may be prohibited by kinetic traps.

Recently, a procedure has been developed by one of us to synthesize bowl-shaped colloidal particles. ${ }^{17}$ This method starts with the preparation of highly uniform silicone oil-inwater emulsion droplets by hydrolysis and polymerization of dimethyldiethoxysilane. Subsequently, the silicone oil droplets were used as templates around which a solid shell with tunable thickness is grown by using tetraethoxysilane. 

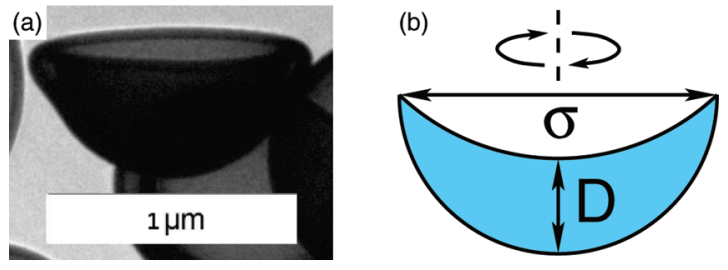

FIGURE 1. (a) Transmission electron micrograph of a colloidal bowl shaped particle. (b) The theoretical model of the colloidal bowl is the solid of revolution of a crescent around the axis as indicated by the dashed line. The thickness of the double-walled bowl is denoted by $D$ and the diameter of the bowl by $\sigma$.

In the next step of the synthesis, the silicone oil in the droplets is dissolved in ethanol, and finally, during drying in air, the shells collapse into hemispherical double-walled bowls (Figure 1). Axially symmetric bowls are found if the ratio of shell thickness to particle radius is between 0.05 and $0.25{ }^{17,18}$ We also note that recently solid hemispherical particles were synthesized at an air-solution interface ${ }^{19}$ and on a substrate. ${ }^{20}$

In our simulations, we model the particles as the solid of revolution of a crescent (see Figure 1). This model particle captures the most important features of the colloidal bowls, a depression and a hemispherical outer shape. The diameter $\sigma$ of the particle and the thickness $D$ are defined as indicated in Figure 1. We define the shape parameter of the bowls by a reduced thickness $D / \sigma$, such that the model reduces to infinitely thin hemispherical surfaces for $D / \sigma=0$ and to solid hemispheres for $D / \sigma=0.5$. The advantage of this simple model is that it interpolates continuously between an infinitely thin bowl and a hemispherical solid particle, and that we can derive an algorithm that tests for overlaps between pairs of bowls, which is a prerequisite for Monte Carlo simulations of hard-core systems.

Our experimental system consists of hemispherical collapsed shells, which were obtained following the procedure described in ref 17 . The only modification was that the dye, rhodamine B-isothiocyanate (RITC), was incorporated in the shell during the coating step. The radius of the uncoated droplets as determined from static light scattering (SLS) experiments is $520 \mathrm{~nm}$. The thickness of the shell was determined by fitting the SLS data of the coated emulsion droplets with the full Mie solution for the scattering factor of core-shell particles with the core radius kept fixed at 520 $\mathrm{nm}$. The result for the shell thickness was $80 \mathrm{~nm}$. The colloids were dispersed in an index-matched mixture of dimethylsulfoxide and ethanol. A bottomless vial was glued to a coverslip and filled with the suspension and the bowls were allowed to sediment. The gravitational length was estimated to be $l / \sigma_{0}=k_{\mathrm{B}} T / m_{\mathrm{B}} g \sigma_{0} \simeq 10$, where $k_{\mathrm{B}}$ is Boltzmann's constant, $g$ is the gravitational acceleration, $T$ is the temperature, $m_{\mathrm{B}}$ is the buoyant mass of the bowls, and $\sigma_{0}$ $=1200 \mathrm{~nm}$, the diameter of shell before collapse. To avoid layering effects, all confocal microscopy images of the sediment were recorded at least $5 \mu \mathrm{m}$ from the bottom wall. The sediment continued to slowly compact for several weeks, while the particles formed stacks. A typical image is shown in Figure 2a taken after 51 days of sedimentation. We clearly observe that the bowls form stacks as can be recognized by the " $\mathrm{C}$ " shapes that are stacked on top of each other. These stacks are present throughout the whole sample and can have lengths up to 10 particles running in random directions. We also note that many stacks are bent and bifurcated by forming Y-shaped junctions. The dynamics of the bowls has been slowed down dramatically as the density at the bottom of the sample had increased significantly due to sedimentation. The bowl-shaped particles seem to get caged by neighboring particles, which may have prevented them from finding a more ordered thermodynamically stable phase. Even after 234 days, the structure had not changed noticeably compared to the structure as observed after 51 days of sedimentation.

We also compare our experimental results with computer simulations. We perform standard Monte Carlo simulations in the isobaric-isothermal ensemble (NPT). Figure 2b shows a typical configuration of bowl-shaped particles with $D=$ $0.36 \sigma$ at $\mathrm{P} \sigma^{3} / k_{\mathrm{B}} T=100$, displaying similar stacking behavior as observed in the experiments. The pressure of $100 k_{\mathrm{B}} T / \sigma^{3}$ is approximately equal to the pressure at the bottom of the experimental system, which was estimated using the total dry weight of particles. Simulations of hard rods ${ }^{21}$ and hard spheres $^{22}$ under gravity show that the structural and thermodynamic properties at a certain height are identical to those of a bulk system at the corresponding pressure. Therefore, the comparison between the structures as obtained from an isotropic compression in simulations and by sedimentation in experiments is fully justified. We find that the density increases smoothly with pressure during the formation of the stacks, indicating a continuous transition from a low-density fluid with hardly any stacks to a highdensity fluid consisting of wormlike stacks whose lengths increase with pressure. To quantify the length of the stacks, we calculated the stack distribution, both in simulations and in experiments. The probability to find a particle in a stack of size $n, \mathscr{P}_{\text {stack }}(n)=n N_{n} / N$, where $N_{n}$ is the number of stacks of size $n$ and $N$ is the total number of particles, is presented in Figure 2c. We show the experimental results along with the results obtained from simulations of bowls with $D / \sigma=0.2,0.3,0.36$, and 0.4 for $P \sigma^{3} / k_{\mathrm{B}} T=100$. The number of stacks of a certain size in a two-dimensional (2D) confocal image was counted by hand, as the current particle tracking techniques cannot be applied for this new type of particle. To compare the simulation results with the $2 \mathrm{D}$ confocal images, we calculated the stack distribution of the bowls within a slab of thickness $\sigma$. Furthermore, a colloid could be identified with certainty as a single particle in the experiment only when it was visible as a "C" shape (see Figure 2a), which was also included in the cluster criterion in the simulations. As can be seen from Figure $2 \mathrm{c}$, the averaged stack length increases significantly as the reduced thickness $D / \sigma$ decreases. In our theoretical model, the 

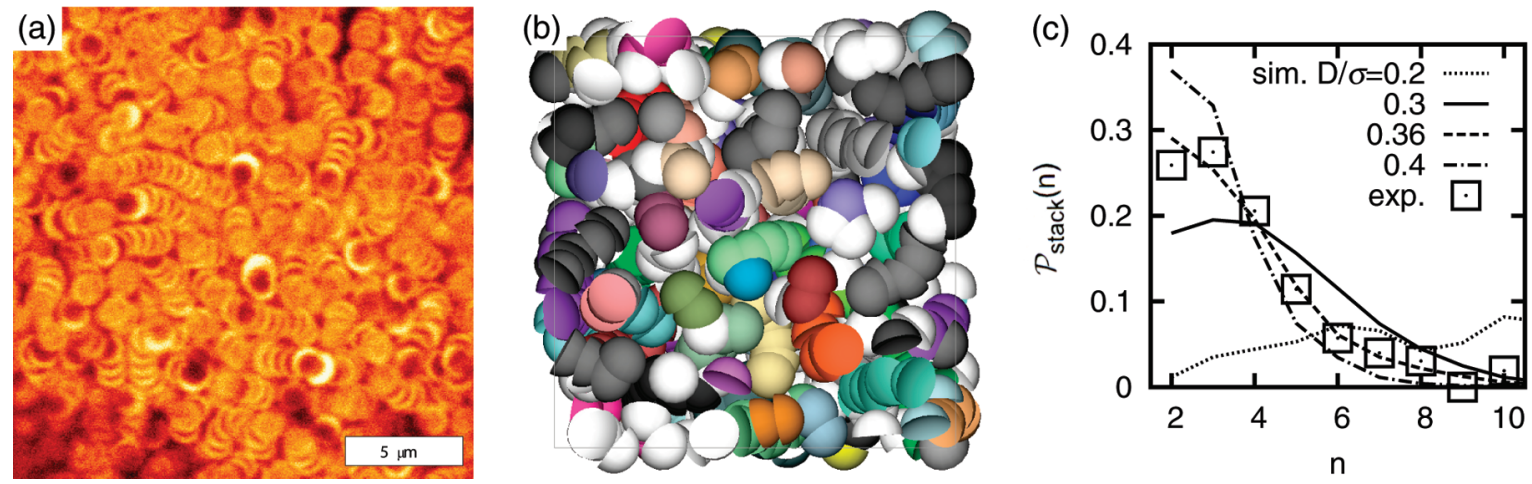

FIGURE 2. (a) Confocal microscope image taken in the sediment formed by the colloidal bowls after 51 days of sedimentation. (b) The final configuration obtained from a simulation at $P \sigma^{3} / k_{\mathrm{B}} T=100$ and $D=0.36 \sigma$. The colors denote different stacks. (c) The probability, $\mathscr{P}_{\text {stack }}(n)$, to find a particle in a stack of size $\mathrm{n}$ in the experiment and in simulations for $D / \sigma=0.2,0.3,0.36$, and 0.4 and pressure $\mathrm{P} \sigma^{3} / k_{\mathrm{B}} T=100$ in a slab (see text). The experimental data are the average of two data sets obtained after 51 and 120 days.

distance of closest approach of two bowls is equal to the thickness $D$ of the bowl-shaped particles. By equating $D$ for the colloidal bowls to the average interparticle distance in a number of short straight stacks, we can map the colloidal bowls onto the theoretical model. In this way, we obtain a reduced thickness $D / \sigma$ of approximately $430 \mathrm{~nm} / \sigma \simeq 0.34$ \pm 0.02 . Here, we have taken the diameter $\sigma$ of the collapsed shell to be $1280 \pm 80 \mathrm{~nm}$, which corresponds to a slight stretching of the outer half-shell to accommodate the inner half-shell. The estimate for $D / \sigma$ is remarkably close to 0.36 , for which we find good agreement for the stack distributions in Figure 2c. Furthermore, we obtained very similar stack distributions in simulations of bowls with slightly modified shapes, provided that the ratio between the distance of closest approach and the diameter was kept constant.

Our simulations also show that the bowls with a thickness $D \geq 0.25 \sigma$ always remained arrested in the wormlike phase, even after long equilibration times, which is similar to the experimental observations. However, for $D / \sigma=0.1$ and 0.2 , we find that the system eventually transforms into a columnar phase in the simulations. This might be explained by the fact that the isotropic-to-columnar transition occurs at lower packing fractions for deeper bowls (smaller $D$ ), which facilitates the rearrangements of particles into stacks and the alignment of the stacks into the columnar phase. However, using our experimental procedure to synthesize colloidal bowls, it is hard to obtain a thickness $D \leq 0.2 \sigma$, as theoretical calculations and experiments show that colloidal shells with smaller shell thickness collapse into bowls with wrinkles, ${ }^{18}$ which make it harder for them to stack.

As preliminary attempts to form ordered phases of the bowl-shaped particles by applying oscillating electric fields (as in ref 23) and sedimentation on a template (as in ref 24) have not been successful, we performed simulations to investigate the equilibrium phase behavior of the bowls. To this end, we first determined candidate crystal structures using a method, which has recently been shown to work very well for a whole range of particle shapes and potentials. ${ }^{25}$ We note that this method is similar in spirit to earlier methods. ${ }^{27,26}$ A system of only $2-6$ particles is simulated using variable box shape Monte Carlo simulations in the NPT ensemble. The pressure is increased by factors of ten until a pressure of $\beta P \sigma^{3}=10^{6}$ is reached. The final configuration was used as the unit cell of a candidate crystal structure, that is, the final configuration of 2-6 particles was periodically repeated to obtain a crystal of several hundreds of particles. Subsequently, we determined the equation of state of the crystal structures in NPT simulations with fully variable box shape. The equation of state of the columnar phase has been measured in a rectangular simulation box, where the box lengths were allowed to change independently. We have used the Einstein integration method as described in ref 28 to determine the Helmholtz free energy of the crystal structures, and we employed the method in ref 29 for the free energy of the columnar phase. The Widom insertion method was used to determine the free energy of the isotropic fluid/wormlike phase at a density close to the isotropic-columnar phase transition. ${ }^{30}$ The free energy as a function of density was determined by integrating the equation of state, ${ }^{31}$ and we employ standard common tangent constructions to determine the packing fractions of the coexisting phases.

In Figure 3, we show the resulting phase diagram in the packing fraction $\phi$-thickness $D / \sigma$ representation. The packing fraction is defined as $\phi=(\pi D / 4)\left(\sigma^{2}-D \sigma+(2 / 3) D^{2}\right) N / V$. We find four different stable crystal phases. In the inverted crystal (IX) and the inverted braid-like crystal (IB), the particles are stacked in columns with half of the columns flipped upside down, such that the rims of the bowls can interdigitate. In the IX, the columns consist of particles that are all aligned head to toe, while in the IB phase, the columns resemble braids with alternating tilt direction of the particles within each column. The solid hemispheres $(D \simeq 0.5 \sigma)$ display two stable crystal structures: the IX' phase can be regarded as a sheared version of IX with alternating orientation of the particles and where the particles are not organized in columns. In the paired face-centered-cubic $\left(\mathrm{fcc}^{2}\right)$ phase, pairs of hemispheres join together to form complete spheres 

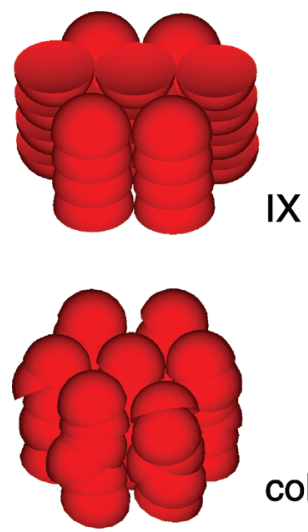

$\mathrm{col}$

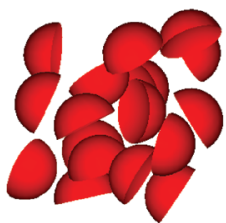

fluid

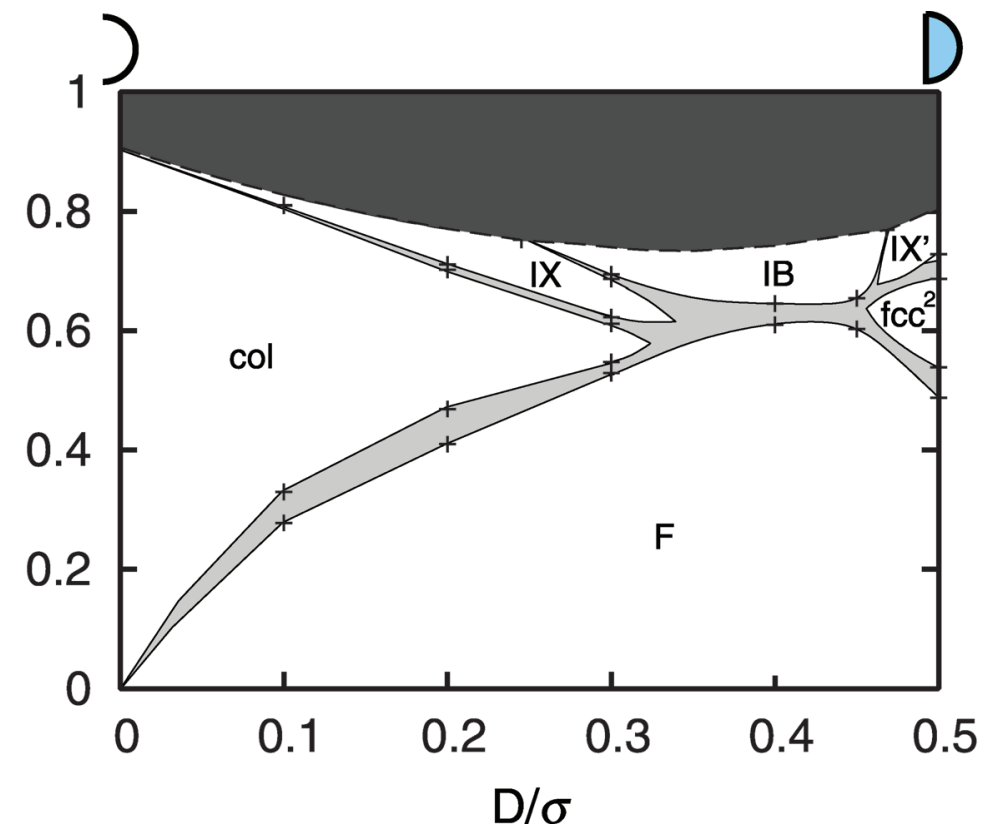

IB

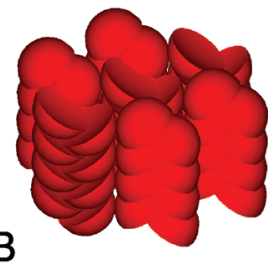

IX'

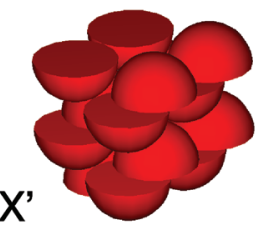

fcc $^{2}$

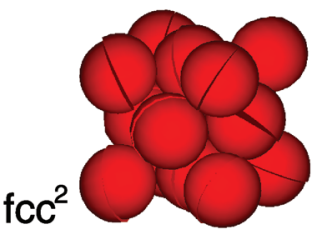

FIGURE 3. Phase diagram of hard bowl-shaped particles in the packing fraction $(\phi)$ versus thickness $(D / \sigma)$ representation. The light gray areas denote the coexistence regions, while the dark gray area indicates the forbidden region as it exceeds the maximum packing fraction of the bowls. The stable crystal phases, IX, IX', IB, and fcc ${ }^{2}$, and the hexagonal columnar phase "col" are drawn schematically on the left and right of the figure. The lines are a guide to the eye.

TABLE 1. Quantitative Description of the Crystalline Phases in the Phase Diagram (Figure 3) ${ }^{a}$

\begin{tabular}{|c|c|c|c|c|}
\hline label & unit cell & space group & $N_{u}$ & remarks \\
\hline IX & orthorhombic & Pmmn & 2 & $\begin{array}{l}\text { The bowl at the corner of the unit cell points along the shortest } \\
\text { lattice vector; the other bowl points in the opposite direction and is } \\
\text { located at the center of the largest face. }\end{array}$ \\
\hline IB & monoclinic & $P 2_{1} / c$ & 4 & $\begin{array}{l}\text { The directions and positions of the particles in the unit cell depend } \\
\text { on } D / \sigma \text { and } \phi \text { in a nontrivial way. }\end{array}$ \\
\hline $\mathrm{IX}^{\prime}$ & hexagonal $^{b}$ & $\mathrm{Cmcm}$ & 6 & $\begin{array}{l}\text { The first three bowls lie in a (nearly) hexagonal plane and point } \\
\text { along one in-plane lattice vector; the other three particles point in } \\
\text { the opposite direction and are located in a different plane but at the } \\
\text { same in-plane positions. }\end{array}$ \\
\hline $\mathrm{fcc}^{2}$ & cubic & & 8 & Each pair of bowls is located at a face centered cubic lattice position. \\
\hline
\end{tabular}

${ }^{a}$ Shown are the label in the phase diagram, the shape of the unit cell, the international short symbol for the space group of each fully aligned crystal at close packing, the number of bowls in the unit cell $N_{u}$, and some remarks describing the structure of the unit cell. The dimensions of the unit cells of the fully aligned crystals vary with aspect ratio $D / \sigma$ and packing fraction $\phi$ in a nontrivial way; therefore we do not list them here.

${ }^{b}$ The IX' unit cell is actually orthorhombic for densities below close packing due to anisotropic expansion.

that can rotate freely on the lattice positions of an fcc crystal. Quantitative descriptions of these four crystals can be found in Table 1 and in the Supporting Information. Note, that the fluid $-\mathrm{fcc}^{2}$ coexistence packing fractions are very close to the packing fractions at coexistence between the fluid $(\phi=$ $0.494)$ and the face center cubic (fcc) crystal ( $\phi=0.545$ ) of hard spheres (the difference is less than 0.01 for both packing fractions). This is caused by the clear similarity between the $\mathrm{fcc}^{2}$ phase of bowls and the fcc phase of spheres.

For $D / \sigma \leq 0.3$, we find an isotropic-to-columnar phase transition at intermediate densities, which resembles the phase diagram of thin hard discs. ${ }^{32}$ However, the fluidcolumnar-crystal triple point for discs is at a thickness-todiameter ratio of about $L / \sigma \sim 0.2-0.3$, while in our case the triple point is at about $D / \sigma \sim 0.3-0.4$. We also find that the columnar phase with all the particles pointing in the same direction is more stable than a columnar phase, where half of the columns are upside down. However, the free energy difference between the two phases is only $\sim 0.01 k_{\mathrm{B}} T$ per particle at $\phi=0.5193$ and $D=0.3 \sigma$. On the basis of this small free energy difference we do not expect polar ordering to occur spontaneously. Similar conclusions, on the basis of direct simulations, were drawn in ref 8. Using this phase diagram, the size ratio ranges for which certain phases are stable can be estimated for collapsed shells, as well as the nanobowls of refs $1-3$ and 5 , by identifying $D$ with the distance of closest approach in a stack of these particles, which can usually be calculated by basic geometry. For instance, it can be shown that half shells obtained by the methods of refs 1,3 , and 5 are likely to form columnar phases $(D / \sigma<0.3)$ when the ratio between the thickness of 
the nanobowls and their inner radius is less than 0.12 , comfortably in the range of thicknesses which can be achieved. $^{3}$

In conclusion, we have studied the phase behavior of hard bowls in Monte Carlo simulations and in experiments. In both systems, we find that the bowls have a strong tendency to form stacks, but the stacks are bent and not aligned. However, for deeper bowls (which are difficult to achieve experimentally with our synthesis route), we find in our simulations spontaneous ordering into a columnar phase. As the experimental system did not show spontaneous ordering, we determined the phase diagram using free energy calculations in computer simulations for a particle shape ranging from an infinitely thin bowl to a solid hemisphere. We find that the columnar phase is stable for $D \leq$ $0.3 \sigma$ at intermediate packing fractions. In addition, we show using free energy calculations that the stable columnar phase possesses polar order. However, the free energy penalty for flipping columns upside down is very small, which makes it hard to achieve complete polar ordering in a spontaneously formed columnar phase of bowls. Our work provides insight in the self-organization of bowl-shaped particles, which might be useful in designing molecular and nanometer-sized bowls.

Acknowledgment. This work was financially supported by the High Potential Programme of Utrecht University and an NWO-VICI grant to M.D.

Supporting Information Available. Lattice parameters of the four crystal phases of bowls. This material is available free of charge via the Internet at http://pubs.acs. org.

\section{REFERENCES AND NOTES}

(1) Wang, X. D.; et al. Nano Lett. 2004, 4, 2223.

(2) Charnay, C.; et al. J. Phys. Chem. B 2003, 107, 7327.

(3) Liu, J.; et al. Adv. Mater. 2005, 17, 1276.

(4) Jagadeesan, D.; et al. Angew. Chem. 2008, 47, 7685.

(5) Love, J. C.; et al. Nano Lett. 2002, 2, 891.

(6) Hosein, I. D.; Liddell, C. M. Langmuir 2007, 23, 8810.

(7) Giersig, M.; Mulvaney, P. Langmuir 1993, 9, 3408.

(8) Ricci, M.; Berardi, R.; Zannoni, C. Soft Matter 2008, 4, 2030.

(9) Rabe, K. M.; et al. In Physics of Ferroelectrics; Springer: Berlin/ Heidelberg, 2007; pp 1-30.

(10) Malthete, J.; Collet, A. J. Am. Chem. Soc. 1987, 109, 7544. (a) Xu, B.; Swager, T. M. J. Am. Chem. Soc. 1993, 115, 1159. (b) Sawamura, M. Nature 2002, 419, 702 .

(11) Rabideau, P. W.; Sygula, A. Acc. Chem. Res. 1996, 29, 235.

(12) Forkey, D. M.; Attar, S.; Noll, B. C.; Koerner, R.; Olmstead, M. M.; Balch, A. L. J. Am. Chem. Soc. 1997, 119, 5766.

(13) Matsuo, Y.; Tahara, K.; Sawamura, M.; Nakamura, E.J. Am. Chem. Soc. 2004, 126, 8725 .

(14) Sakurai, H.; Daiko, T.; Sakane, H.; Amaya, T.; Hirao, T. J. Am. Chem. Soc. 2005, 127, 11580

(15) Kawase, T.; Kurata, H. Chem. Rev. 2006, 106, 5250.

(16) Cinacchi, G.; van Duijneveldt, J. S. J. Chem. Phys. Lett. 2010, 787.

(17) Zoldesi, C. I.; Walree van, C. A.; Imhof, A. Langmuir 2006, 22, 4343.

(18) Quilliet, C.; Riera, C.; van Blaaderen, A.; Imhof, A. Eur. Phys. J. E 2008, 27, 13

(19) Higuchi, T.; Yabu, H.; Shimomura, M. Colloids Surf. A 2006, 284, 250

(20) Xia, Y.; Gates, B.; Li, Z. Adv. Mater. 2001, 13, 409

(21) Savenko, S. V.; Dijkstra, M. Phys. Rev. E 2004, 70, No. 051401.

(22) Marechal, M.; Dijkstra, M. Phys. Rev. E 2007, 75, No. 061404.

(23) van der Zande, B. M. I.; Koper, G. J. M.; Lekkerkerker, H. N. W. J. Chem. Phys. B 1999, 103, 5754.

(24) van Blaaderen, A.; Ruel, R.; Wiltzius, P. Nature 1997, 385, 321.

(25) Filion, L. Phys. Rev. Lett. 2009, 103, 188302.

(26) Buch, V.; Martoňák, R.; Parrinello, M. J. Chem. Phys. 2006, 124, 204705

(27) Schroer, J. W.; Monson, P. A. J. Chem. Phys. 2000, 112, 8950.

(28) Marechal, M.; Dijkstra, M. Phys. Rev. E 2008, 77, No. 061405.

(29) Bates, M. A.; Frenkel, D. Phys. Rev. E 1998, 57, 4824.

(30) Widom, B. J. Chem. Phys. 1963, 39, 2808.

(31) Frenkel, D.; Smit, B. Understanding molecular simulation; Academic Press: San Diego, 2002

(32) Veerman, J. A. C.; Frenkel, D. Phys. Rev. A 1992, 45, 5632. 